\title{
AN AD HOC WIRELESS SYSTEM FOR SMALL TOWNS AND RURAL AREAS
}

\author{
F. L. Figueiredo, M. A. de Siqueira, M. C. de Castro, H. J. de Souza, A. L. Pacifico, L dos Santos \\ and J. A. Martins
}

\begin{abstract}
Although the huge progress in Telecommunications area in the last years, part of Brazilian population still does not have access to any communication system. In order to provide a solution for this scenario, $\mathrm{CPqD}$ is developing a system based on Ad Hoc wireless network to provide voice and data services for this part of Brazilian population. This paper describes the estimated market, benefits and requirements of this system. Reference architecture model and protocol architecture are proposed for Ad Hoc wireless network. It is also described a testbed, which has been designed for Ad Hoc network performance evaluation.
\end{abstract}

Keywords - Ad Hoc, wireless network, testbed

\section{INTRODUCTION}

Although the huge progress in mobile communications and data transmission areas in the last years, Brazil still has people that do not have access to any telecommunication system. This part of Brazilian population lives in small towns or rural areas, where the deployment costs of telecommunications networks are high, which is one of the reasons for the current scenario.

One of the advantages of Ad Hoc wireless networks is that there is no need for high cost infrastructure, and so, the deployment of these systems is cheaper than traditional telecommunication solutions. Thus, a system based on Ad Hoc wireless network can be an alternative to provide telecommunications services to the referred market.

Considering this issue, $\mathrm{CPqD}$ is developing a system based on Ad Hoc wireless network that shall provide voice and low data transmission rate services and shall be tuned to the needs and characteristics of Brazilian market. Another relevant requirement for this system is the development of low cost user terminals.

The system being developed consists of:

- Ad Hoc user terminal and other network elements

- Ad Hoc Network Management System

- Ad Hoc Wireless network planning methodology

- Economical and regulatory analysis of services for Ad Hoc wireless networks

Considering the Brazilian regulatory model for telephony services, this Ad Hoc system could be employed as [1]:

The authors work by $\mathrm{CPqD}$ - Centro de Pesquisa e Desenvolvimento em Telecomunicações

fabricio@cpgd.com.br:

mcastro@cpqd.com.br;

albertol@cpqd.com.br; jansouza@cpqd.com.br; lsantos@cpqd.com.br;
- Support for Public Switched Telephone Network (PSTN) services

- Support for Digital Communication Service, which is being defined by the Brazilian Regulatory Agency.

- Support for Limited Services

The next sections describe some results obtained during the project development. In section 2 a market survey is presented. The system requirements are described in section 3. Section 4 presents a reference architecture model for a system based on Ad Hoc wireless network. The testbed implemented is described in section 5 and the conclusion of this work is presented at section 6 .

\section{MARKET}

In order to estimate the potential market for a system based on Ad Hoc wireless network, CPqD conducted a market survey in 2003. In this work, a questionnaire was answered by people living in small towns and rural areas in different regions of Brazil that do not have access to Public Switched Telephone Network.

The following topics were analyzed:

- Availability of Telecommunication systems

- Market for telecommunication services

- Interest of people in having access to telecommunications systems, considering regions that do not have any telecommunication system available.

The results showed that $50 \%$ of interviewed people use alternative systems and the most used is cellular system $(69 \%)$. However, they are not satisfied with the systems and services provided, mainly due to the following reasons:

- High cost

- Bad coverage area (loss of signal, interference)

- Data transmission rates are very low

Furthermore, these users can be considered as potential candidates to use a system that do not have these problems.

On the other hand, $50 \%$ of interviewed people do not use any kind of telecommunication system. They are a very attractive market for a system based on Ad Hoc wireless networks. In fact, $82 \%$ of these users stated that they need a communication system and their main interest is for voice services.

Considering the costs due to system deployment, people intend to pay values ranging from $R \$ 200.00$ to $R \$ 1000.00$ and they would like to spend $\mathrm{R} \$ 50.00$ to $\mathrm{R} \$ 90.00$ monthly with the services.

In conclusion, there is a market in Brazil for a telecommunication system that provides voice and low data transmission rate services at low cost. 


\section{SYSTEM REQUIREMENTS}

The main requirements of the system based on Ad Hoc wireless network being developed are:

- It shall provide voice and data services.

- The user data transmission rate should be greater than $64 \mathrm{kbit} / \mathrm{s}$.

- It shall use low cost user terminals.

- It should provide connection to PSTN and IP networks.

- It shall be based on packet transmission.

- It shall be able to identify system faults and to provide external visualization of them.

- It shall be possible to collect data for quality of service evaluation.

- It shall operate at frequency bands according Brazilian spectrum regulation.

- It shall operate in multihop scenarios.

\subsection{ROUTING PROTOCOL REQUIREMENTS}

The routing protocols used by wired networks are not appropriated to Ad Hoc networks mainly due to the characteristics of these networks, such as dynamic topology, bandwidth constraint and time-varying characteristics of wireless links.

The requirements of routing protocols for Ad Hoc networks are [2] [3]:

- Distributed routing - it is more fault tolerant than centralized routing and involves less overhead.

- It shall be loop free.

- It shall have security mechanisms.

- It shall have mechanisms to detect dropped links

- It shall support route longevity.

- It shall have mechanisms to manage packet loss.

- It shall be able to provide the quality of service demanded by the application.

- It must optimally use the scarce resources of wireless Ad Hoc networks.

- It must converge to optimal routes.

- It shall $\mathrm{d}$ be able to recover routes in a quick and efficient way.

- It shall select routes according quality of service necessary for the application.

The routing protocols that have been studied and simulated in this project are: Dynamic Source Routing (DSR), Destination Sequenced Distance-Vector Routing Protocol (DSDV), Ad Hoc On-Demand Distance-Vector Routing Protocol (AODV), Ad Hoc On-Demand Multipath Distance-Vector Routing Protocol (AOMDV), Optimized Link State Routing (OLSR), Associativity-Based Routing $(A B R)$ and Cluster Based Routing Protocol (CBRP).

\subsection{MAC PROTOCOL REQUIREMENTS}

Due to specific characteristics of wireless Ad Hoc networks, such as absence of central control unit and multihop operation, the MAC protocols should present the following requirements [2] [3] [4]:
- Control overhead shall be kept as low as possible

- It shall be scalable to large networks.

- It shall ensure that all nodes are treated fairly with bandwidth allocation.

- It shall present good performance in multihop scenarios.

- It shall minimize the exposed and hidden nodes problem in order to improve the network throughput.

- It shall grant channel access to nodes in such a way that collisions are minimized.

- It shall support security mechanisms.

- It shall support mechanisms to detect dropped links.

- It shall support mechanisms to manage packet loss.

- It shall support mechanisms for power control in order to decrease the interference and minimize the power consumption.

The studied and simulated MAC protocols include Carrier Sensing Multiple Access with Collision Avoidance (CSMA/CA), Media Access Protocol for Wireless LANs (MACAW), Multiple Access with Collision Avoidance Protocol (MACA), Multiple Access with Collision Avoidance with Piggy-Backed Reservation (MACA/PR) and Multiple Access with Collision Avoidance by Invitation (MACA-BI).

\subsection{FREQUENCY BAND CONSIDERATIONS}

The choice of system operation frequency band should take into account the mean distance between user terminals, which can vary from $300 \mathrm{~m}$ in urban environments until 20 $\mathrm{km}$ in rural areas.

Considering the spectrum regulation in Brazil, the following frequency bands should be considered [5] [6] [7] [8]:

- $400 \mathrm{MHz}$ band: $406,10-413,05 \mathrm{MHz}$ and $423,05-$ $430 \mathrm{MHz}$

- $\quad 900 \mathrm{MHz}$ band: $902-907,5 \mathrm{MHz}$ and $915-928 \mathrm{MHz}$

- $\quad 2400 \mathrm{MHz}$ band: $2400-2483,5 \mathrm{MHz}$

- $\quad 5000 \mathrm{MHz}$ band: $5275-5780 \mathrm{MHz}$

The use of $400 \mathrm{MHz}$ band seems to be very appropriated, because the transmitted power can be greater than 10 Watts, resulting in a covering area of $20 \mathrm{~km}$ radius. However, this band is not adequate for operation in urban environments due to interference generated by vehicle ignition noise. The $900 \mathrm{MHz}, 2400 \mathrm{MHz}$ and $5000 \mathrm{MHz}$ bands are more suited for operation in urban environments while the $400 \mathrm{MHz}$ band is more attractive for rural areas.

\section{NETWORK TOPOLOGY AND ARCHITECTURE}

Taking into account the system requirements, different deployment scenarios and kind of services, a network topology was specified [2], and it is shown at Figure 1. 


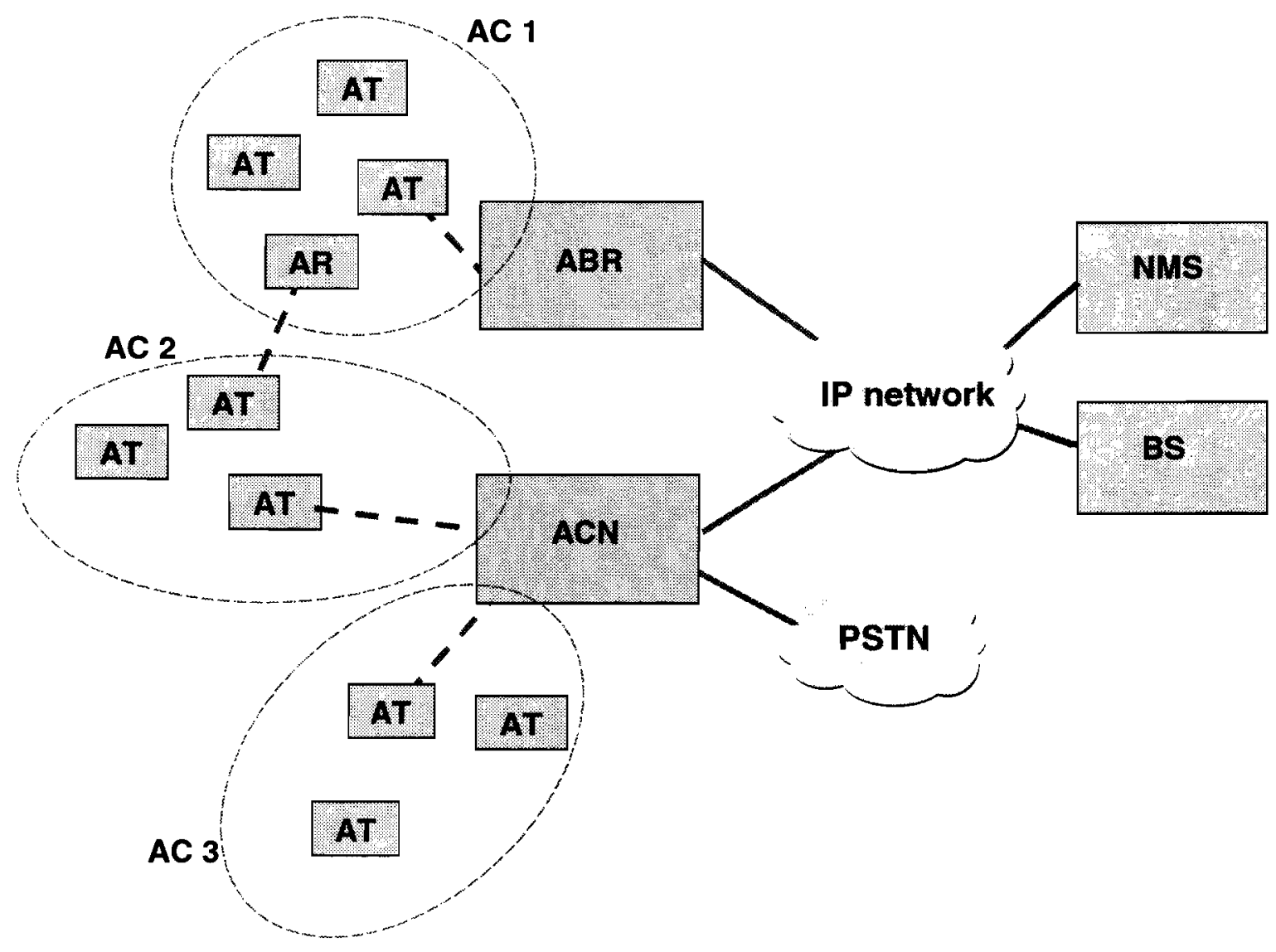

Figure 1: System topology.

The physical elements of the topology are:

- Ad Hoc Cluster (AC)

- Ad Hoc Core Network (ACN)

- Ad Hoc Border Router (ABR)

- Ad Hoc Terminal (AT)

- Ad Hoc Repeater (AR)

- Ad Hoc Network Management System (NMS)

- Ad Hoc Billing System (BS)

The Ad Hoc Cluster is a set of the following elements:

- Ad Hoc Terminal

- Ad Hoc Repeater

- Ad Hoc Border Router

- Ad Hoc Core Network

These elements are interconnected via air interface.

The Ad Hoc Terminal is the device employed by users to access the system services.

The Ad Hoc Core Network e (ACN) is responsible for the connection between an $\mathrm{Ad}$ Hoc Cluster and other networks such as PSTN and IP networks.

The Ad Hoc Border Router is used for connection between an Ad Hoc Cluster and IP networks.

The Ad Hoc Repeater is employed for increasing the coverage area of an Ad Hoc Cluster.
The Network Management System is responsible for the network management.

The Billing system is responsible for billing functions.

Considering the network topology, a reference architecture model is proposed [2], as shown at Figure 2.

This reference model allows different implementations, and can be partly implemented.

Each architecture physical element is composed of a set of logical elements, which are described in the next sections.

\subsection{AD HOC CORE NETWORK (ACN)}

The Ad Hoc Core Network is responsible by connection between Ad Hoc Clusters and other networks, such as PSTN and IP networks. It performs the user registration and authentication and call establishment procedures.

The Ad Hoc Core Network architecture is shown at Figure 3 and it consists of the following logical elements:

- Core Service Node (CSN)

- Voice Server (VS)

- Voice Gateway (VG)

- Authentication and Authorization Server (AAA)

- Address Sever (AS)

- $\quad$ Short Message Server (SMS)

- Name Server (NS)

- Border Router (BR) 


\subsubsection{VOICE SERVER (VS)}

All the Ad Hoc Core Network elements are

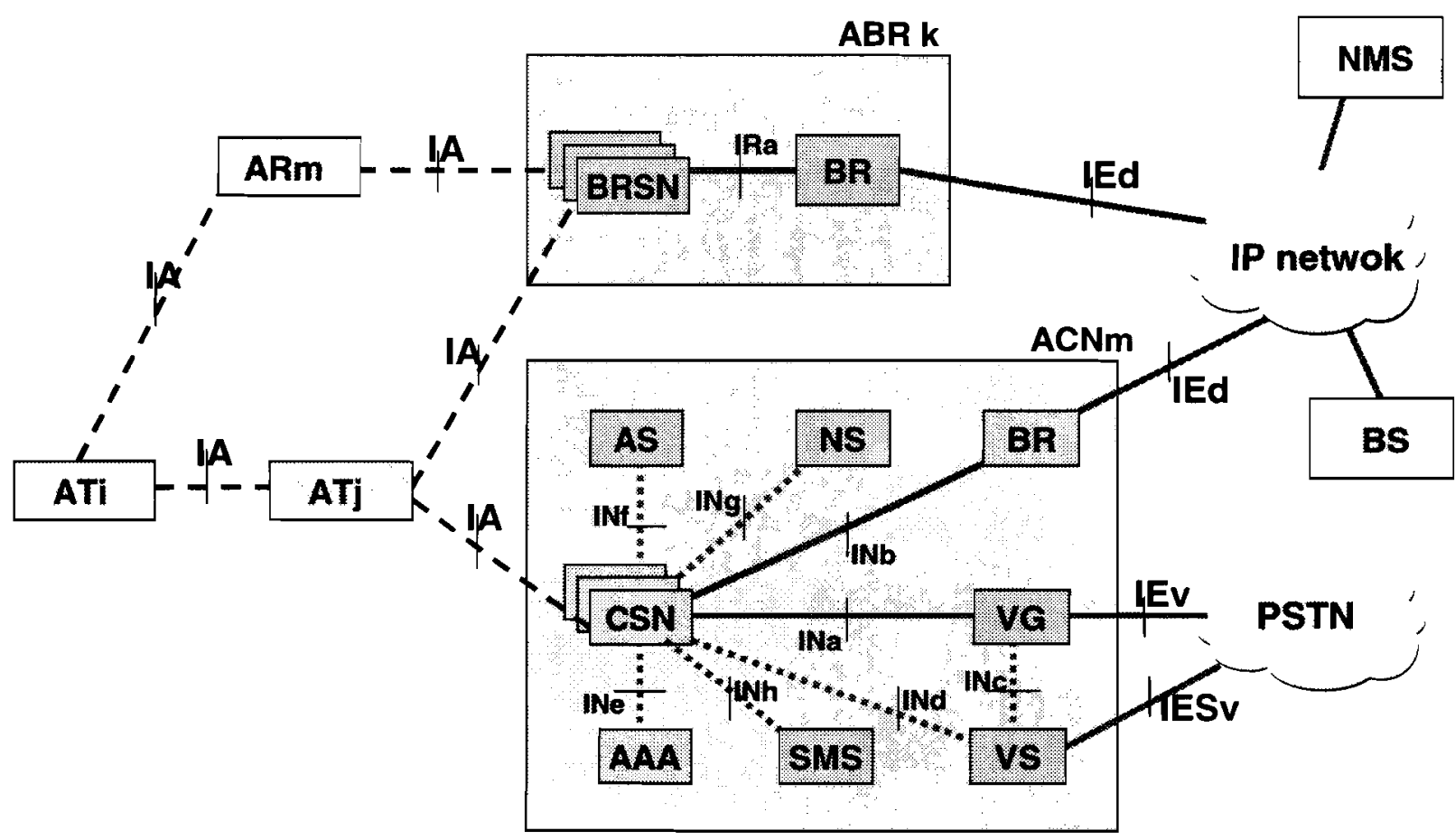

Figure 2: Reference architecture model.

interconnected using IP protocols.

\subsubsection{CORE SERVICE NODE (CSN)}

The Core Service Node is responsible for connection between an Ad Hoc Cluster and Ad Hoc Core Network logical elements.

The main features of the Core Service Node are:

- Communication with Ad Hoc Terminals: Core Service Node supports the same radio (physical layer) and MAC protocols than Ad Hoc Terminals.

- Packets forwarding: Core Service Node forwards the packets received from an Ad Hoc terminal according to a defined route.

- Routing: the Core Service Node establishes and maintains the routes to Ad Hoc Terminals in the same Ad Hoc Cluster.

- Routes redistribution: the Core Service Node should enables the connection between Ad Hoc Core Network internal nodes and external nodes with no need for address translation.

- Packets Filtering: the Core Service Node controls the packets forwarding, based on static rules for headers comparison.

- Addresses Mapping: the Core Service Node maps one network address to another network address, enhancing the process for messages routing or retransmission.

The Ad Hoc Core Network may support several Core Service Nodes.
The Voice Server controls the calls in the network and records all call details (Call Details Record - CDR). It is also responsible for controlling the Voice Gateway.

\subsubsection{VOICE GATEWAY (VG)}

The Voice Gateway connects the Ad Hoc network to the PSTN and it is also responsible for voice coding and decoding.

\subsubsection{AUTHENTICATION AND AUTHORIZATION SERVER (AAA)}

It is responsible for the access control to system resources and services and for system security.

The basic functions of this server are:

- Authentication: it identifies the subscriber based on username and password.

- Authorization: it authorizes the subscriber to access the system services.

- Accounting: consists of the registration and accounting of the system resources usage by the subscribers.

\subsubsection{ADDRESS SERVER (AS)}

The Address Server is responsible for IP addresses distribution to Ad Hoc Terminals and Ad Hoc Core Network logical elements. 


\subsubsection{NAME SERVER (NS)}

The Name Server performs names mapping into IP addresses.

\subsubsection{SHORT MESSAGES SERVER (SMS)}

Short Messages Server manages and storages short messages within the Ad Hoc Cluster.

\subsubsection{BORDER ROUTER (BR)}

The Border Router is responsible for interconnection of an Ad Hoc Core Network to IP networks, allowing access to Internet and Ad Hoc Border Routers.

It performs address mapping and packets filtering functions.

\subsection{AD HOC BORDER ROUTER (ABR)}

The Ad Hoc Border Router is responsible for interconnection between Ad Hoc Cluster and IP networks.

It comprises the following logical elements:

- Border Router Service Node (BRSN);

- Border Router (BR)

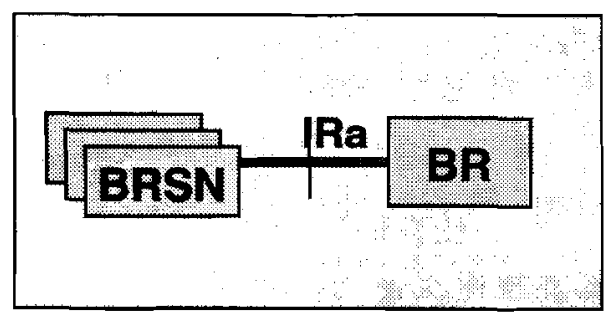

Figure 4: Ad Hoc Border Router architecture.

\subsubsection{BORDER ROUTER SERVICE NODE (BRSN)}

The Border Router Service Node is responsible for interconnection between Ad Hoc Cluster and Border Router.

It performs a subset of the previously defined functionalities for the Ad Hoc Core Network, as follows:

- Packets forwarding

- Routing

- Packets Filtering

- Addresses Mapping

- Routes redistribution

\subsubsection{BORDER ROUTER (RB)}

The Border Router is responsible for interconnection between the Ad Hoc Border Router and an Ad Hoc Core Network via an external packet data network.

It also performs address mapping and packets filtering functions.

\subsection{AD HOC TERMINAL (AT)}

The Ad Hoc Terminal is the user equipment, which allows the access to the system services.

\subsection{AD HOC REPEATER (AR)}

The Ad Hoc Repeater function is to retransmit the received signal in order to extend the Ad Hoc Cluster coverage.

\subsection{INTERFACES}

The interfaces between the architecture elements are:

- IA: air interface between elements TA, AR, ACN and $A B R$

- INa: interface between elements NSN and VG (within ACN)

- INb: interface between elements NSN and BR (within ACN)

- INc: interface between elements VG and VS (within $\mathrm{ACN}$ )

- INd: interface between elements NSN and VS (within ACN)

- Inez: interface between elements NSN and AAA (within $\mathrm{ACN}$ )

- INf: interface between elements NSN and AS (within ACN)

- INg: interface between elements NSN and NS (within $\mathrm{ACN}$ )

- INh: interface between elements NSN and SMS (within ACN)

- IEd: interface between $\mathrm{ACN}$ and packet data networks

- IEv: interface between ACN (VG) and PSTN for voice transport

- IESv: interface between ACN (VS) and PSTN for voice call control

- IRa: interface between elements BRSN and BR (within ABR)

\subsection{PROTOCOL ARCHITECTURE}

The protocol architecture comprises the protocol stack defined for each element in the reference architecture model.

\subsubsection{TRANSMISSION PLANE}

The transmission plane is a hierarchical protocol structure that supports the transmission of information between Ad Hoc Terminals and external networks, for voice and data services. This protocol stack includes all necessary procedures to guarantee the data delivery, such as packet retransmission, packet flows control, error detection and correction and others. 


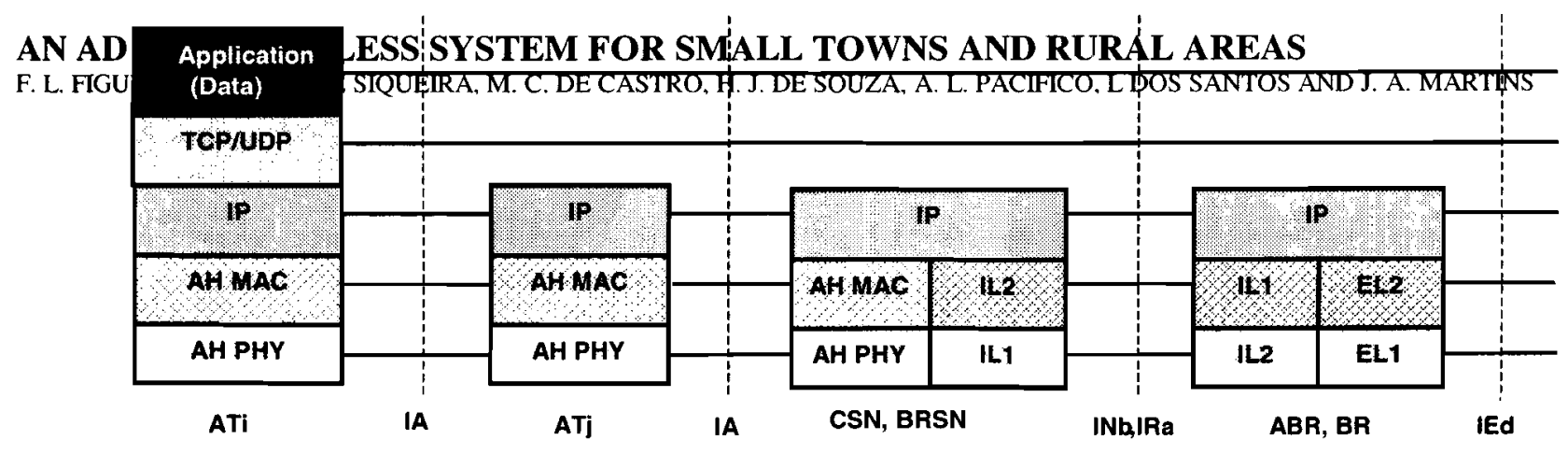

Figure 5: Transmission Plane for data services.

The transmission plane for data services is illustrated at Figure 5. In this plane, the Ad Hoc terminal ATi establishes a multihop connection to an external network, using intermediate Ad Hoc terminals (terminal ATj) to reach an Ad Hoc Core Network or Ad Hoc Border Router, that are responsible for interconnection to external data networks.

The terminal ATi transmission plane comprises the following layers:

- Ad Hoc PHY (AH PHY): physical layer Ad Hoc

- Ad Hoc MAC (AH MAC): link layer defined for Ad Hoc network

- IP: routing protocol

- TCP/UDP: transport protocol

- Application (data): data services supported by Ad Hoc system.

For a given connection, the intermediate terminals present only the layers AH PHY, AH MAC and IP.

The air interface IA is used for connection between $\mathrm{Ad}$ Hoc terminals and between the terminal ATj and the Core Service Node.

The layers ILI (Internal Layer 1) and IL2 (Internal Layer 2) are respectively generic physical and link layers appropriated for IP packets transport. The Core Service Node connects to Border Router via interface INb.

The interface IEd is responsible for connection between the Border Router and external data networks, based on or Ad Hoc Border Router, that are responsible for interconnection to PSTN.

The terminal ATi transmission plane for voice services comprises the layers:

- $\quad$ Ad Hoc PHY (AH PHY): physical layer

- Ad Hoc MAC (AH MAC): link layer defined for Ad Hoc network:

- IP: routing protocol;

- TCP/UDP: transport protocol

- RTP: Session protocol for VoIP services

- Application (Voice): voice service supported by the Ad Hoc system.

For a given call, the intermediate terminals presented only the layers AH PHY, AH MAC and IP.

The air interface IA is used for connection between $\mathrm{Ad}$ Hoc terminals and between terminal $\mathrm{ATj}$ and Core Service Node.

The interface IE is used for connection between the Voice Gateway and PSTN.

\subsubsection{SIGNALING PLANE}

The Signaling plane consists of signaling protocols that support the transmission plane functionalities, including network access control, network resources management, supplementary services and others.

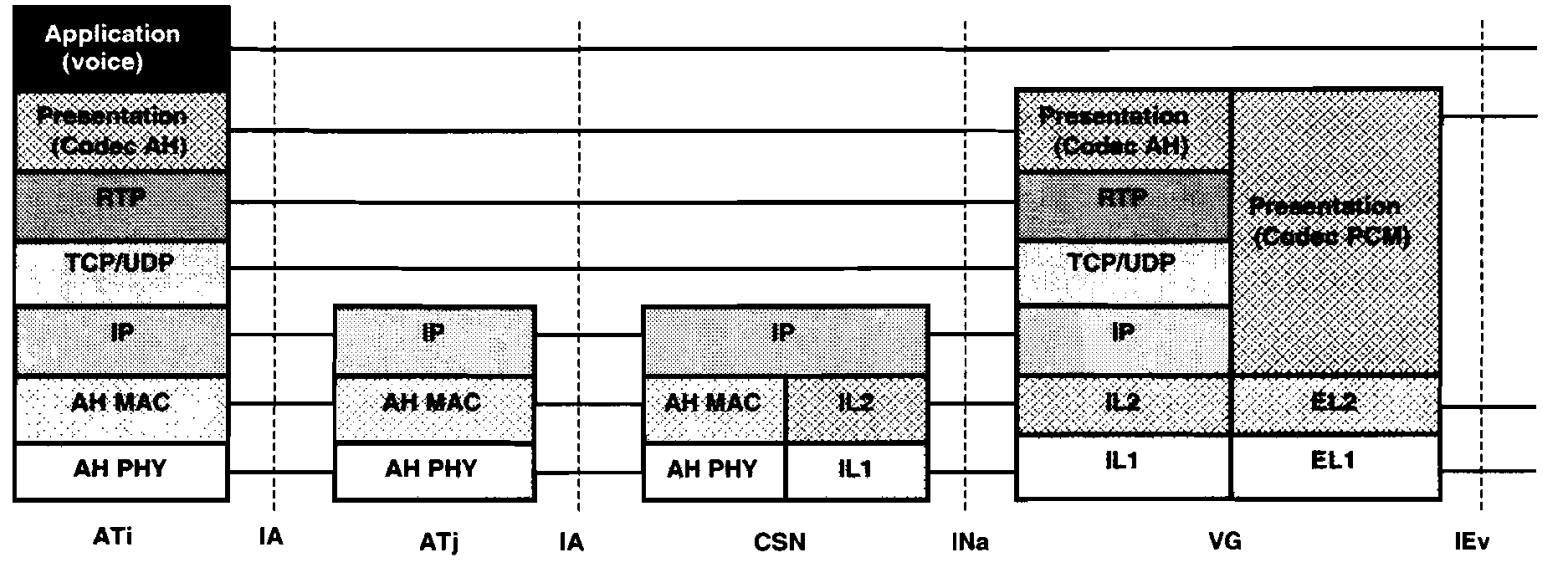

Figure 6: Transmission plane for voice services.

layers EL1 (External Layer 1) and EL2 (External Layer 2).

The physical layer may be an El trunk.

The transmission plane for voice services is illustrated at Figure 6. In this plane, the Ad Hoc terminal ATi establishes a multihop connection to PSTN, using intermediate Ad Hoc terminals (terminal ATj) to reach an Ad Hoc Core Network 


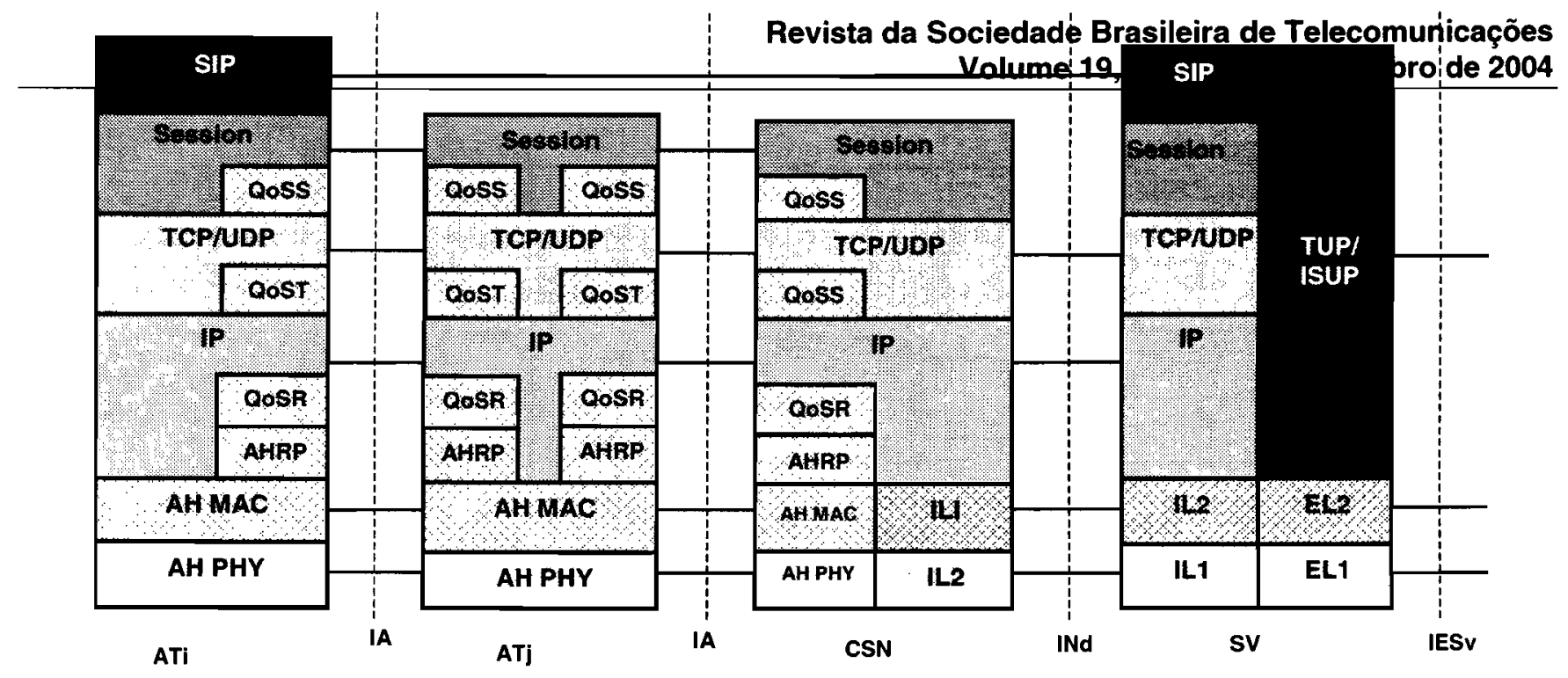

Figure 7: Signaling plane for call control using SIP protocol.

The Signaling plane for voice call control using SIP protocol is shown at Figure 7.

The physical layer (AH PHY) and link layer (AH MAC) are the same an in the Transmission plane. The application layer at terminals $\mathrm{ATi}$ and $\mathrm{ATj}$ and Core Service Node corresponds to SIP protocol.

The sublayer Ad Hoc Routing Protocol (AHRP) performs the network routing function.

The quality of service (QoS) management functions are implemented by a framework composed of sublayers distributed in different layers of signaling protocol stack.

The sublayers are the following:

- QoS Routing (QoSR): it is responsible for QoS monitoring and control of active routes in each node, selecting and maintaining routes based on
- QoS Session (QoSS): it acts at session layer and it manages the QoS level in each established traffic session.

The air interface IA is used for connection between Ad Hoc terminals and between terminal $\mathrm{ATj}$ and Core Service Node.

The interface INd is used for connection between Core Service Node and Voice Server.

The interface IESv is responsible for call control signaling exchange between Ad Hoc Core Network and PSTN. This signaling corresponds to protocols TUP/ISUP.

The signaling plane to support the Domain Resolution functionality and Short Message Service is illustrated at Figure 8.

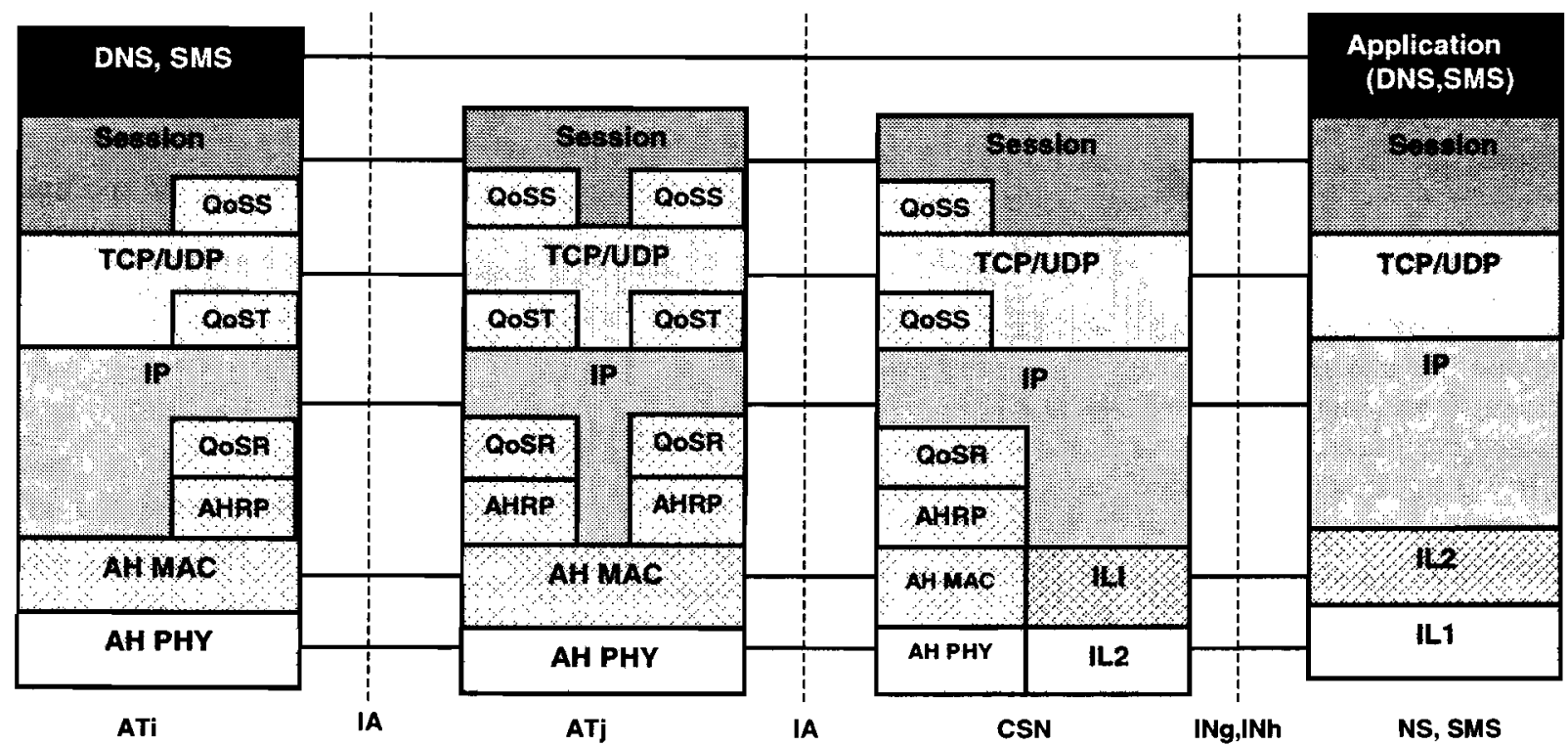

Figure 8: Signaling plane to support the Domain resolution functionality and Short Message Service.

QoS requirements for each service

- QoS Transport (QoST): it acts at transport layer, adapting TCP and UDP protocols to provide the QoS levels necessary for data and voice services 


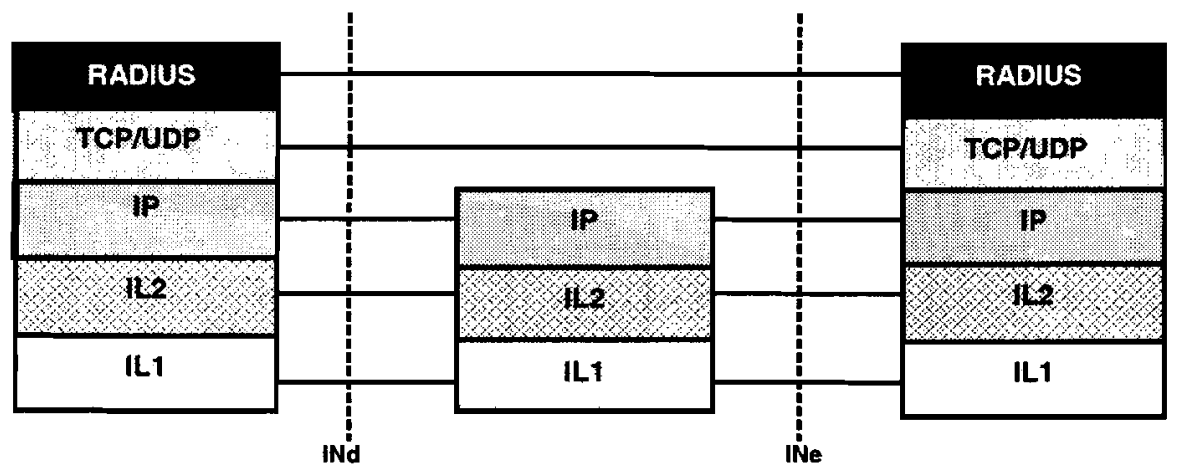

Figure 9: Signaling plane for Radius protocol.

The Signaling plane to support the Radius protocol is shown at Figure 9. This protocol is implemented at application layer and performs authentication, authorization and accounting functions.

The Signaling plane to support the MGCP protocol is shown at Figure 10. The protocol was implemented at application layer and it is used by Voice Server to control the Voice Gateway.

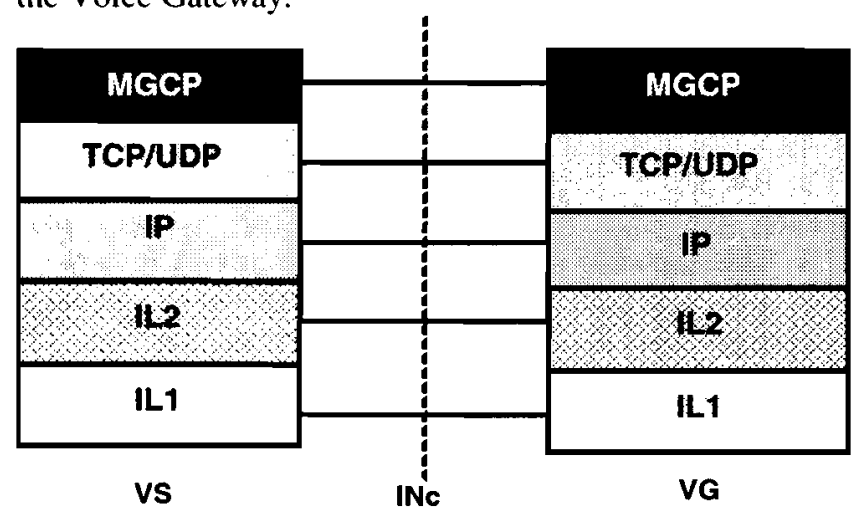

Figure 10: Signaling plane for protocol MGCP.

\section{TESTBED}

In order to evaluate the performance of different routing protocols and to analyze IEEE $802.11 \mathrm{~b}$ configuration parameters [9], it was implemented a testbed [10].

The testbed comprises Ad Hoc user terminals and an IP gateway. It was implemented in indoor environment and it supports voice and data calls between the testbed elements in a multihop way.

\subsection{NODES DESCRIPTION}

The nodes were built using off-the-shelf hardware.

Each node consists of a computer (notebook or desktop) equipped with a WLAN card and some nodes are also equipped with a telephone. The WLAN card is used for communication between the testbed nodes.

The node performing the gateway function is equipped with an Ethernet NIC (Network interface card) for connecting to an IP network.
The operating system running on the computers is Linux Red Hat 9 kernel 2.4.26 and the routing protocol implemented runs at user level and does not require any special configuration of operating system.

\subsubsection{HARDWARE}

The hardware used in the testbed was:

- Intel Pentium $41.5 \mathrm{GHz}$ computers with $256 \mathrm{MB}$ of RAM (desktop)

- Intel Pentium $42.66 \mathrm{GHz}$ computers with $256 \mathrm{MB}$ of RAM (notebook)

- WLAN Cisco Aironet 350 PCI IEEE 802.1 l b card

- Ethernet 3Com 3c590 board

- Digium TDM 400P

\subsubsection{RADIO AND MAC}

The radio functionalities were implemented using WLAN cards with the following characteristics:

- Standard IEEE 802.11b

- Frequency band: $2.4 \mathrm{GHz}$

- Channel number: 11

- Operation mode: Ad Hoc

- Physical layer: Direct sequence spread spectrum

- Medium Access: Carrier Sensing Multiple Access with Collision Avoidance (CSMA/CA)

- Transmission rate: $11 \mathrm{Mbps}$

- Transmission power: $20 \mathrm{dBm}$

\subsubsection{ROUTING PROTOCOL}

The routing protocol adopted for the tesbed id the Optimized Link State Routing (OLSR), according the standard RFC 3626 of IETF [12].

This protocol is an optimized version of the classic Link state protocol [11] and it is a proactive routing protocol. The link state forward mechanism employed by OLSR is called multipoint relaying and it reduces the control overhead. In this mechanism, each node maintains a subset of neighbors called multipoint relays (MPR) that are responsible for forwarding the link state packets.

Every node in the network stores information about onehop and two-hop neighbors, which is periodically updated based on messages broadcasted by the nodes. 
To provide connection with other networks, one node in the network can be declared gateway using the messages

- Route 2: node 1 - node 2 - node 3 - node 4 - 3 hops

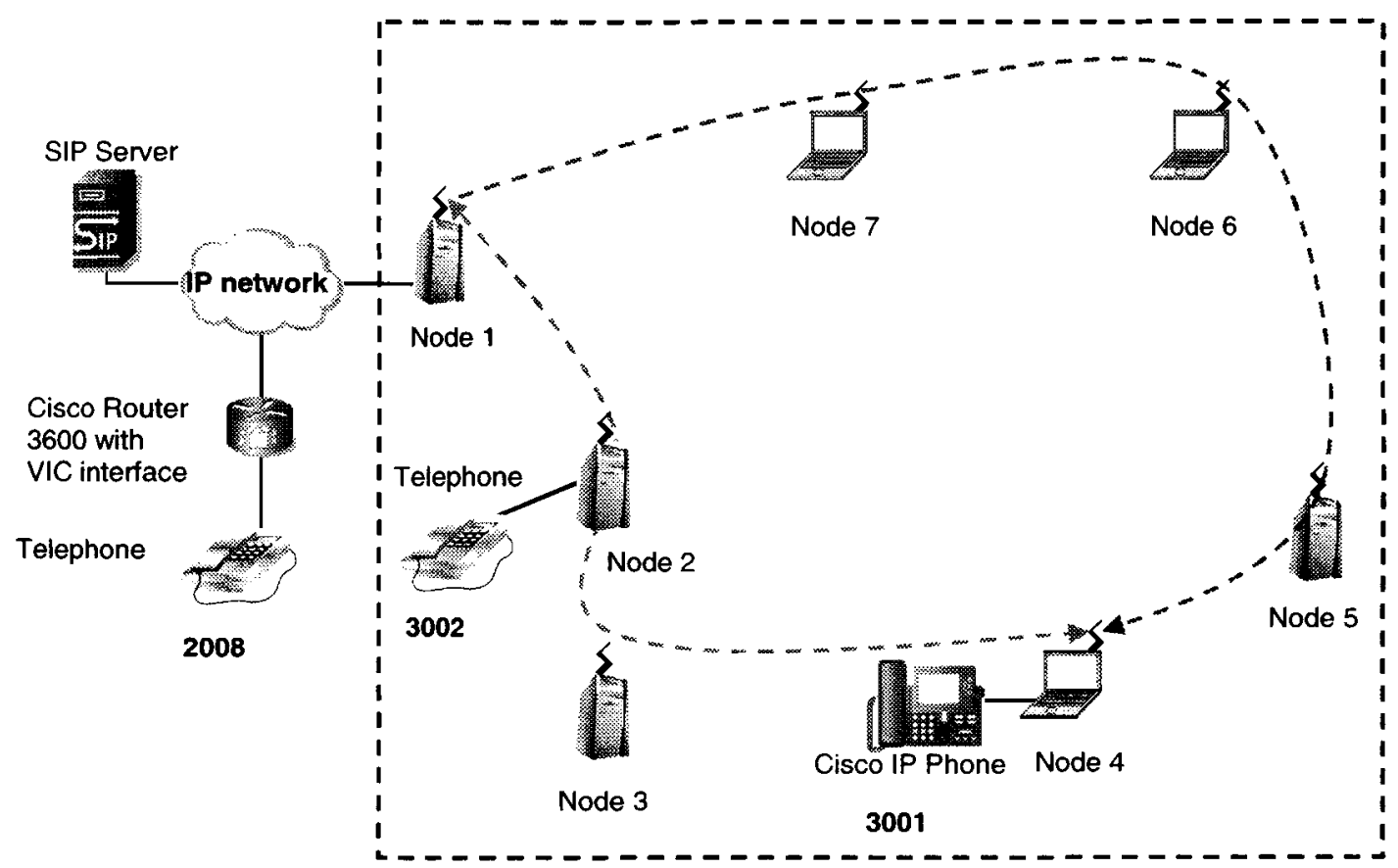

Figure 11: Testbed topology.

Host and Network Association (HNA).

\subsection{TESTBED TOPOLOGY}

The testbed topology consists of seven nodes, as shown at Figure 11.

It was deployed inside of $\mathrm{CPqD}$ installations.

The description of the testbed nodes is presented at Table 1.

\begin{tabular}{|c|c|}
\hline Node & Function \\
\hline 1 & Ad Hoc terminal and gateway to router \\
\hline 2 & $\begin{array}{c}\text { Ad Hoc terminal with voice card and analog } \\
\text { telephone }\end{array}$ \\
\hline 3 & Ad Hoc terminal \\
\hline 4 & Ad Hoc terminal with VoIP telephone \\
\hline 5 & Ad Hoc terminal \\
\hline 6 & Ad Hoc terminal \\
\hline 7 & Ad Hoc terminal \\
\hline
\end{tabular}

Table 1: Testbed nodes

The equipment identified as nodel is connected to an IP network, providing the connection between the Ad hoc wireless network and the IP network.

The signaling protocol used to establish the calls is Session Initiation Protocol (SIP), defined in IETF RFC 2543.

To establish a call from user number 2008 to user number 3001 , there are two possible routes, with different number of hops:

- Route 1: node 1 - node 7 - node 6 - node 5 - node 4 -4 hops

\subsection{TESTS RESULTS}

The tests consisted of establishment of voice and data calls and evaluation of voice quality and latency. For some calls, after their establishment, it was forced a route change.

The following qualitative results were obtained:

- The voice quality was good in the majority of calls and it was possible to establish a conversation.

- Increasing the interference level near the terminal, the conversation was interrupted but the call was not dropped. The duration of interruption period was directly dependent of interference level.

- When a route change was forced, the conversation was interrupted for a period of approximately 5 seconds and after this the conversation was reestablished with the same quality as before.

There are on course tests to evaluate other routing protocols and WLAN configuration parameters, considering the Ad Hoc network performance.

\section{CONCLUSION}

In this paper was presented the requirements of a system based on Ad Hoc wireless network to provide voice and low data rate services to small towns and rural area in Brazil.

It was described a reference architecture model for Ad Hoc wireless network and a testbed implemented for routing protocol performance evaluation. 
The preliminary test results of the implemented testbed showed that an Ad Hoc system can be used for data and voice communication services.

This system based on Ad Hoc wireless systems can improve the Digital inclusion in Brazil, providing voice and data services to Brazilian population that does not have access to any telecommunication system.

Moreover, it can contribute to improve the competition at Brazilian telecommunications market.

\section{BIBLIOGRAFY}

[1] F. Basseto, "Objectives and requisites of an Ad Hoc access networks," (in Portuguese), version AA, PD.30.11.67A.0025A/RT-01-AA. Campinas, CPqD, Ago/2004, Technical Report.

[2] F. L. Figueiredo, M. A. de Siqueira, H. P. de B. Pimentel, D. Fernandes, A. Paganuchi, F. M. F. Rocha, "Reference architecture of an Ad Hoc network," (in Portuguese), version AA. PD.30.1 1.69A.0025A/RT-02-AA. Campinas, CPqD, Jun/2004, Technical Report.

[3] C. S. R. Murthy, B. S. Manoj, "Ad Hoc Wireless Networks - Architectures and Protocols", 2004, Prentice Hall.

[4] P. Cardieri, M. D. Yacoub, E. J. Leonardo, "State of the art of medium access control techniques in Ad Hoc multihop systems", (in Portuguese) version AA, PD.30.11.69A.0027A/RT-01-AA. Campinas, CPqD, Apr/2004, Technical Report.

[5] F. M. Filho, "RF device analysis available for application in Ad Hoc", (in Portuguese) version AA, PD.30.11.69A.0025A/RT-03-AA. Campinas, CPqD, Apr/2004, Technical Report.

[6] Norm $n^{\circ} 07 / 97$ "Channelization and use conditions of frequencies for digital radiocommunication systems in the $400 \mathrm{MHz}$ band with transmission capacity of up to $8 \mathrm{Mbit} / \mathrm{s}$," (in Portuguse), Anatel

[7] Annex to Resolution $n^{\circ} 169$, of october 5th, 1999, Channelization and use conditions of frequencies for digital radiocommunication systems in the $400 \mathrm{MHz}$ band with transmission capacity of up to $8 \mathrm{Mbit} / \mathrm{s}$," (in Portuguese), Anatel

[8] Annex to Resolution $\mathrm{n}^{\circ} 305$, of July 26th, 2002, "Regulation of restrict radiation radiocommunication equipment,"(in Portuguese), Anatel

[9] ANSI/IEEE Std 802.11, 1999 Edition. Part 11: Wireless LAN Medium Access Control (MAC) and Physical Layer (PHY) Specifications.

[10] A. L. Pacífico, L dos Santos, "Description of the implemented lab prototype," version AA, PD.30.11.69A.0025A/RT-06-AA. Campinas, CPqD, Ago/2004, Technical Report.

[11] J. Moy. OSPF Version 2, in IETF RFC 1583, 1994

[12] T. Clausen and P. Jacquet. "RFC3626 - Optimized Link State Routing Protocol (OLSR)", category: experimental, October 2003.
Fabrício Lira Figueiredo received his BS in electrical engineering from the Federal University of Pernambuco, at Recife, Brazil, in 1995. He received MS degree in electrical engineering from State University of Campinas, at Campinas, Brazil, in 1999. $\mathrm{He}$ is currently a researcher at Centro de Pesquisa e Desenvolvimento em Telecomunicações (CPqD), which is a research and development center in telecommunications area. located at Campinas. He is working on his $\mathrm{PhD}$ thesis at State University of Campinas and his research focuses on design, analysis and control of wireless networks. Current interests include: ad hoc wireless networks, wireless local and metropolitan area networks, capacity an QoS in wireless networks, wireless sensor networks, cellular networks, mobile applications, digital signal processing and speech recognition.

Marcos Antônio de Siqueira is currently a Network Engineer at $\mathrm{CPqD}$ Telecommunications Research and Development Center where is conducting research on Internetworking issues including mobile ad hoc networks and emerging Internet protocols. Prior to this, he worked in several projects related to IP networks design, installation, configuration, and management in Brazilian telecommunication companies. Marcos received the B.S. Degree in Electrical Engineering from the Federal University of Goiás (UFG), Brazil (1999), and the M.Sc. Degree in Computer Engineering from the State University of Campinas (Unicamp), Brazil (2002). Currently, he is a Ph.D. student in the Department of Computer Engineering and Industrial Automation at the State University of Campinas (FEEC/UNICAMP), Campinas, Brazil.

Marcel Cavalcanti de Castro received his B.S. degree in Electrical Engineering from Federal University of Goiás, Brazil, in 2000. Currently, he is a MSc student in the Department of Telematics at State University of Campinas and works as a Telecom Engineer at CPqD Telecom \& IT Solutions. His current interests include Ad hoc Wireless Network, Quality of Service and Next Generation Networks.

Henrique Januário de Souza received his B.S. degree in Electrical Engineering from State University of Campinas, 1978. From 1979 to 1994 he worked as an R\&D engineer at CPqD in PDH and SDH systems. In 1995 he joined a group of wireless systems in $\mathrm{CPqD}$ and since 2002 is involved in research activities of ad hoc networks. His interests are in the development of MAC protocols applied in ad hoc terminals.

Alberto Lucizani Pacifico received the B.S. degree in Electrical Engineering from State University of Campinas, in 2000. Since January of 2001 he has worked at $\mathrm{CPqD}$ in research and development activities at wireless communication area. Currently, he is working with ad hoc wireless networks and his current activities include ad hoc terminals tests and routing protocols and WLAN performance evaluation.

Leandro dos Santos is a technical assistant and student in Electrical Engineering of UNISAL. Since 2003 he is working at $\mathrm{CPqD}$ in research activities at ad hoc wireless networks. His current activities include ad hoc terminals tests and routing protocols and WLAN performance evaluation.

José Antonio Martins received the B.S degree in Electrical Engineering from University of São Paulo, in 1985, the MSc. degree in Electrical Engineering from State University of Campinas, in 1991 and the $\mathrm{Ph}$. D. degree from State University of Campinas, in 1997. Currently, he is working at CPqD, where he is a research engineer at wireless communications area. His current 
interests include ad hoc wireless network, radio resources management, wireless systems and digital signal processing. 